\title{
Convergence of Monte Carlo algorithm for solving integral equations in light scattering simulations
}

\author{
MACIEJ KRASZEWSKI, JERZY PLUCIŃSKI* \\ Department of Metrology and Optoelectronics, \\ Faculty of Electronics, Telecommunications and Engineering, \\ Gdańsk University of Technology, Gabriela Narutowicza 11/12, 80-233 Gdańsk, Poland \\ ${ }^{*}$ Corresponding author: pluc@eti.pg.edu.pl
}

\begin{abstract}
The light scattering process can be modeled mathematically using the Fredholm integral equation. This equation is usually solved after its discretization and transformation into the system of algebraic equations. Volume integral equations can be also solved without discretization using the Monte Carlo algorithm, but its application to the light scattering simulations has not been sufficiently studied. Here we present the implementation of this algorithm for one- and three-dimensional light scattering computations and discuss its applicability in this field. We show that the Monte Carlo algorithm can provide valid and accurate results but, due to its convergence properties, it might be difficult to apply for problems with large volumes or refractive indices of scattering objects.
\end{abstract}

Keywords: light scattering, Monte Carlo, numerical methods.

\section{Introduction}

The numerical methods for simulating light scattering process can be divided into two groups: the wave methods that operate directly on the Maxwell equation or the Helmholtz equation and the radiative transfer methods that are based on the radiative transfer equation (RTE) treating lights propagation more like the incoherent energy transfer than the propagation of the coherent wave. The first group contains such methods as the finite -difference simulation [1], the method of moments (MoM) [2- 2$]$, the discrete dipole approximation (DDA) [ $\underline{3}-\underline{5}]$, or the T-matrix method [ $\underline{6}]$. The best-known method from the second group is probably the Monte Carlo (MC) algorithm for solving the radiative transfer equation which has been successfully applied to many different problems connected to the light scattering [ㄱ-13].

The MC solver for RTE allows to simulate the propagation of light in macroscopic volumes of scattering media such as biological tissue. However, it does not allow for direct treatment of the phenomena connected to the wave nature of the light such as 
interference or diffraction. Several propositions of modification of this approach appeared [ $\underline{13}-15]$ but still simulation of propagation of the coherent light beams in macroscopic volumes of scattering media is still an open problem.

The MC approach itself can be applied not only to the RTE but also for different types of integral equations. For light scattering problems, the Helmholtz equation, which describes the propagation of the electromagnetic wave, can also be transformed into the integral equation $[\underline{2}-\underline{4}, \underline{16}]$ and thus solved with the proper MC algorithm $[\underline{17}, \underline{18}]$. The usage of such an approach was proposed by Pereira and SHERIF [19]. Also, in our previous paper, we applied a similar technique to solve the system of linear equations for discretized Rayleigh scattering simulations [20].

The interesting part of the algorithm described in [19] is that it directly solves the underlying integral equation without any discretization. This makes it suitable for dealing with very complex geometries for which the discretization process might be difficult or inaccurate. Also, the authors of [19] claim that the MC algorithm can be used to simulate the scattering of the coherent light beams in large (comparing to the wavelength) volumes of the scattering medium. However, to the best of our knowledge, the further development of this method has not been presented by any research group. Also, the paper [19] presents only an idea of the numerical method and not its implementation for the light scattering problem. Therefore, the behavior and applicability of the $\mathrm{MC}$ algorithm for light scattering simulations were not properly studied.

In this paper, we present an analysis of the convergence of the $\mathrm{MC}$ algorithm in case of one- and three-dimensional light scattering simulations. We show that the convergence issue is the crucial factor that prohibits the use of the $\mathrm{MC}$ algorithm for volumes much larger than the wavelength of the light. We also present an idea to improve the convergence properties of the algorithm by using the approach similar to preconditioning systems of linear algebraic equations.

\section{Description of Monte Carlo algorithm}

\subsection{Description of light scattering process with the Fredholm integral equation}

The propagation of the light in an inhomogeneous medium can be described by an equation that can be derived directly from the Maxwell equations [21]:

$$
\nabla^{2} \mathbf{E}(\mathbf{r})+k_{0}^{2} n^{2}(\mathbf{r}) \mathbf{E}(\mathbf{r})+\nabla(\ln (\mu(\mathbf{r}))) \times \nabla \times \mathbf{E}(\mathbf{r})+\nabla(\mathbf{E}(\mathbf{r}) \cdot \nabla(\ln (\varepsilon(\mathbf{r}))))=\mathbf{0}
$$

where $\mathbf{E}(\mathbf{r})$ is the spatial distribution of the complex amplitude of the electric field, $\mathbf{r}$ is the position vector, $k_{0}$ is the wave number in a vacuum, $\varepsilon(\mathbf{r})$ is the dielectric constant, $\mu(\mathbf{r})$ is the magnetic permeability, $n(\mathbf{r})$ is the refractive index of the propagating medium, and $\mathbf{0}$ is the zero vector. 
In particular, if $\nabla(\ln (\varepsilon(\mathbf{r})))$ and $\nabla(\ln (\mu(\mathbf{r})))$ are negligibly small, we obtain the well-known Helmholtz equation

$$
\nabla^{2} \mathbf{E}(\mathbf{r})+k^{2}(\mathbf{r}) \mathbf{E}(\mathbf{r})=\mathbf{0}
$$

where $k(\mathbf{r})=k_{0} n(\mathbf{r})$ is the wave number in the medium.

In the one-dimensional case for isotropic materials, Eq. (2) can be transformed into an integral form $[\underline{16}, \underline{21}]$

$$
E(x)=E^{\mathrm{inc}}(x)+\int_{-\infty}^{\infty} G\left(x, x^{\prime}\right) U\left(x^{\prime}\right) E\left(x^{\prime}\right) \mathrm{d} x^{\prime}
$$

where $E^{\mathrm{inc}}(x)$ is the incident electric field that fulfills the homogeneous Helmholtz equation in a vacuum, $U(x)=k_{0}^{2}\left(n^{2}(x)-1\right)$ is the so-called scattering potential, and $G\left(x, x^{\prime}\right)=G(X)$, where $X=x-x^{\prime}$, is the Green's function [22]

$$
G(X)=\frac{i \exp (i k|X|)}{2 k}
$$

In Eq. (3), the electric field vector from Eq. (2) was replaced by the scalar values since for one-dimensional problems Eq. (2) for two orthogonal polarization states of the light are decoupled and can be solved independently.

In the three-dimensional problem, the following integral equation can be derived [ $\underline{3}-\underline{4}]$ :

$$
\mathbf{E}(\mathbf{r})=\mathbf{E}^{\mathrm{inc}}(\mathbf{r})+\int_{V} \overleftrightarrow{\mathbf{G}}\left(\mathbf{r}, \mathbf{r}^{\prime}\right) U\left(\mathbf{r}^{\prime}\right) \mathbf{E}\left(\mathbf{r}^{\prime}\right) \mathrm{d}^{3} \mathbf{r}^{\prime}
$$

where $V$ is the volume of the scattering object and $\overleftrightarrow{\mathbf{G}}\left(\mathbf{r}, \mathbf{r}^{\prime}\right)=\overleftrightarrow{\mathbf{G}}(\mathbf{R})$, where $\mathbf{R}=\mathbf{r}-\mathbf{r}^{\prime}$, is the dyadic Green's function given by

$$
\overleftrightarrow{\mathbf{G}}(\mathbf{R})=\frac{\exp (i k R)}{4 \pi R}\left[\left(\overleftrightarrow{\mathbf{I}}-\frac{\mathbf{R} \otimes \mathbf{R}}{R^{2}}\right)-\frac{1-i k R}{k^{2} R^{2}}\left(\overleftrightarrow{\mathbf{I}}-\frac{3 \mathbf{R} \otimes \mathbf{R}}{R^{2}}\right)\right]
$$

where $R=|\mathbf{R}|, \mathbf{R} \otimes \mathbf{R}$ is a dyadic defined by vector $\mathbf{R}$, and $\overleftrightarrow{\mathbf{I}}$ is an identity dyadic.

Equation (5) describes the electric field $\mathbf{E}(\mathbf{r})$ as the sum of two components: $\mathbf{E}^{\text {inc }}(\mathbf{r})$ and $\int_{V} \overleftrightarrow{\mathbf{G}}\left(\mathbf{r}, \mathbf{r}^{\prime}\right) U\left(\mathbf{r}^{\prime}\right) \mathbf{E}\left(\mathbf{r}^{\prime}\right) \mathrm{d}^{3} \mathbf{r}^{\prime}$. Further, we will denote these quantities as the total field, the incident field, and the scattered field, respectively.

\subsection{Monte Carlo algorithm for solving the Fredholm integral equation}

The integral form of the Helmholtz equation is a basis for such numerical methods as the method of moments or the discrete dipole approximation [2- $\underline{5}]$. In these methods, the entire volume of the light scattering object is divided into small subvolumes. Then 
it is assumed that both scattering potential and the electric field inside each subvolume are constant and the underlying integral equation is discretized and transformed into the system of linear algebraic equations. These equations can then be solved using various numerical algorithms.

Equation (3) or (5) can also be solved in a different way. There is a well-known Monte Carlo algorithm for solving directly the Fredholm integral equation such as Eq. (3) or (5) that does not require any discretization or assumption of the electric field being piecewise constant [17-19]. We describe the MC algorithm by starting with expanding the Eq. (5) into the Neumann series

$$
\begin{aligned}
\mathbf{E}(\mathbf{r})= & \mathbf{E}^{\mathrm{inc}}(\mathbf{r})+\int \overleftrightarrow{\mathbf{G}}\left(\mathbf{r}, \mathbf{r}^{\prime}\right) U\left(\mathbf{r}^{\prime}\right) \mathbf{E}^{\mathrm{inc}}\left(\mathbf{r}^{\prime}\right) \mathrm{d}^{3} \mathbf{r}^{\prime} \\
& +\iint \mathbf{G}\left(\mathbf{r}, \mathbf{r}^{\prime}\right) U\left(\mathbf{r}^{\prime}\right) \mathbf{G}\left(\mathbf{r}^{\prime}, \mathbf{r}^{\prime \prime}\right) U\left(\mathbf{r}^{\prime \prime}\right) \mathbf{E}^{\mathrm{inc}}\left(\mathbf{r}^{\prime \prime}\right) \mathrm{d}^{3} \mathbf{r}^{\prime} \mathrm{d}^{3} \mathbf{r}^{\prime \prime}+\ldots
\end{aligned}
$$

Proposed MC algorithm requires the following input:

- Parameters of the scattering problem: $k, \mathbf{E}^{\mathrm{inc}}(\mathbf{r}), U(\mathbf{r})$, and $V$.

- Maximal path length: $L_{\max }$.

- A number of iterations of the algorithm: $N_{\mathrm{it}}$.

The algorithm consists of the following steps:

- Select positions for which $\mathbf{E}(\mathbf{r})$ needs to be evaluated: $\mathbf{r}_{1}^{\mathrm{e}}, \mathbf{r}_{2}^{\mathrm{e}}, \ldots, \mathbf{r}_{j}^{\mathrm{e}}$.

- For $i$ in the range from 1 to $j$ :

$\circ \mathbf{E}\left(\mathbf{r}_{i}^{\mathrm{e}}\right) \leftarrow 0$.

- Repeat $N_{\text {it }}$ times:

- Select initial position $\mathbf{r}_{0}$ by random sampling from the uniform probability distribution over the entire volume of the scattering object.

$\circ \mathbf{w} \leftarrow \mathbf{E}^{\text {inc }}\left(\mathbf{r}_{0}\right)$.

- Select path length $L$ as a random integer from range from 0 to $L_{\max }$ with every value having the same probability of being selected.

- For $l$ in the range from 1 to $L$ :

- Select position $\mathbf{r}_{l}$ by random sampling from the uniform probability distribution over the entire volume of the scattering object.

- $\mathbf{w} \leftarrow V U\left(\mathbf{r}_{l-1}\right) \overleftrightarrow{\mathbf{G}}\left(\mathbf{r}_{l}, \mathbf{r}_{l-1}\right) \mathbf{w}$.

- For $i$ in the range from 1 to $j$ :

- $\mathbf{E}\left(\mathbf{r}_{i}^{\mathrm{e}}\right) \leftarrow \mathbf{E}\left(\mathbf{r}_{i}^{\mathrm{e}}\right)+\frac{L_{\max }+1}{N_{\text {it }}} V U\left(\mathbf{r}_{l}\right) \overleftrightarrow{\mathbf{G}}\left(\mathbf{r}_{i}^{\mathrm{e}}, \mathbf{r}_{l}\right) \mathbf{w}$.

The main difference between the above MC algorithm and the classical approach to solving the volume integral equation for light scattering problem (e.g. the DDA method) is the fact that the MC algorithm does not require discretization of the equation or the scattering object itself (Fig. 1). This might be reflected in its accuracy and ability to handle various complex geometries.

The extension of the above algorithm for the one-dimensional case is straightforward. One needs to replace quantities $\mathbf{E}$ and $\overleftrightarrow{\mathbf{G}}$ by their scalar forms and use the length 
a

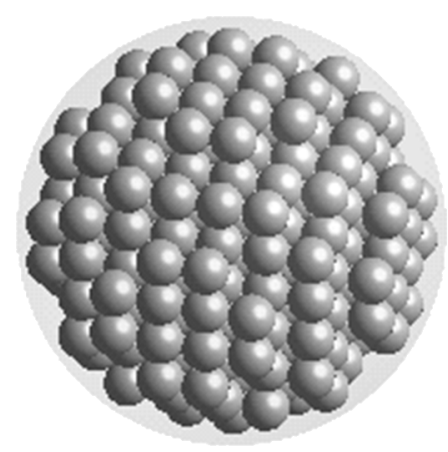

b

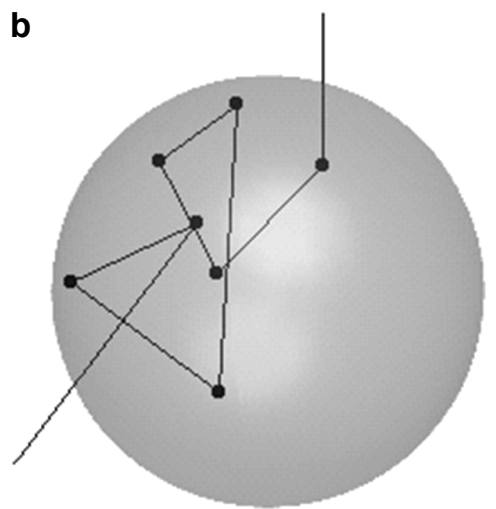

Fig. 1. Difference between the classical approach to analyzing light scattering problem using volume integral equations (a) compared to the presented Monte Carlo algorithm (b). In a classical approach, the integral equation is discretized by dividing the scattering object into small subvolumes and transformed into the system of algebraic equations. In the presented Monte Carlo algorithm, the scattering object is not discretized and the integral equation is solved by sampling its volume by the random processes.

of the scattering object instead of its volume. Also, note that there is an important difference in the MC algorithm described above and the algorithms described in [17-19]. In our algorithm, the path length is limited to the value $L_{\max }$ while typically presented algorithms do not directly limit the path length. This causes our implementation to provide the biased estimator of the electric field but allows us to study how the number of terms in Neumann expansions influences the accuracy of the results.

The MC algorithm described in the previous section can be easily modified to calculate the electric field distribution in the far-field zone. In the final step of the algorithm, the term $\overleftrightarrow{\mathbf{G}}\left(\mathbf{r}_{i}^{\mathrm{e}}, \mathbf{r}_{l}\right)$ should be replaced by its far-field approximation

$$
\overleftrightarrow{\mathbf{G}}\left(\mathbf{s}, \mathbf{r}_{l}\right) \approx \frac{\exp \left(-i k \mathbf{s} \cdot \mathbf{r}_{l}\right)(\overleftrightarrow{\mathbf{I}}-\mathbf{s} \otimes \mathbf{s})}{4 \pi R_{0}}
$$

where $\mathbf{s}$ is the unit vector in the scattered wave propagation direction and $R_{0}$ is the distance between the scattering particle and the observer.

Usually, in case of far-field distribution, it is more useful to operate on the normalized amplitude of the scattered field that does not decrease with distance from the observer. In such a case, the Green's function from Eq. (8) needs to be multiplied by $R_{0}$.

\subsection{Convergence of the Neumann expansion for light scattering problems}

In this section, we discuss the problem of convergence of the numerical method described in Sections 2.1 and 2.2. Note that by convergence we do not mean the convergence of the MC estimators $\tilde{\mathbf{E}}_{j}(\mathbf{r})$ of the Neumann expansions but rather the convergence of the 
expansion itself, i.e. whether adding additional terms of the expansion leads to the decrease in the estimator bias.

It is known that the Neumann expansion of the Fredholm integral equation of the second kind in the form

$$
f(\mathbf{r})=b(\mathbf{r})+\int_{V} K\left(\mathbf{r}-\mathbf{r}^{\prime}\right) f\left(\mathbf{r}^{\prime}\right) \mathrm{d}^{n} \mathbf{r}^{\prime}
$$

where $\mathbf{r} \in V, n$ is the number of problem dimension, for given continuous kernel function $K\left(\mathbf{r}-\mathbf{r}^{\prime}\right)$ and for given function $b(\mathbf{r})$, is convergent if the spectral radius (i.e. the largest module of all eigenvalues) of the integral operator $\hat{\mathbf{K}}$ is defined as

$$
\hat{\mathbf{K}} f(\mathbf{r})=\int_{V} K\left(\mathbf{r}-\mathbf{r}^{\prime}\right) f\left(\mathbf{r}^{\prime}\right) \mathrm{d}^{n} \mathbf{r}^{\prime}
$$

and is smaller than 1 [17].

Since it might be difficult to calculate the spectral radius of the operator $\hat{\mathbf{K}}$, one can use the stronger criterion [19]

$$
\int_{V}|K(\mathbf{r})| \mathrm{d}^{n} \mathbf{r}<1
$$

In case of the problem of one-dimensional light scattering from a thin layer, Eq. (11) leads to the condition

$$
\frac{L}{\lambda}<\left(\pi\left(n_{\mathrm{s}}^{2}-1\right)\right)^{-1}
$$

where $L$ is the thickness of the layer, $\lambda$ is the wavelength in a vacuum, and $n_{\mathrm{s}}$ is the refractive index of the layer.

In case of three-dimensional scattering, the integral from Eq. (11) is a dyadic that makes the analysis less straightforward. For this reason, we studied the convergence properties for the scalar three-dimensional Green's function for a spherical particle, which leads to the condition

$$
k^{2}\left(n_{\mathrm{s}}^{2}-1\right) \int_{0}^{2 \pi} \int_{0}^{\pi} \int_{0}^{R}(4 \pi r)^{-1} r^{2} \sin \theta \mathrm{d} r \mathrm{~d} \theta \mathrm{d} \varphi<1
$$

or

$$
\frac{R}{\lambda}<\left(2 \pi^{2}\left(n_{\mathrm{s}}^{2}-1\right)\right)^{-1 / 2}
$$

where $R$ is the radius of the particle, $\lambda$ is the wavelength in a vacuum, $n_{\mathrm{s}}$ is the refractive index of the sphere, $r, \theta$, and $\varphi$ are the spherical coordinates in the spherical coordinate system.

The maximal values of $L / \lambda$, as well as of $R / \lambda$ as a function of $n_{\mathrm{s}}$ are plotted in Fig. 2 . For relatively small values of the refractive index $n_{\mathrm{s}}$, the maximal thickness of the thin 

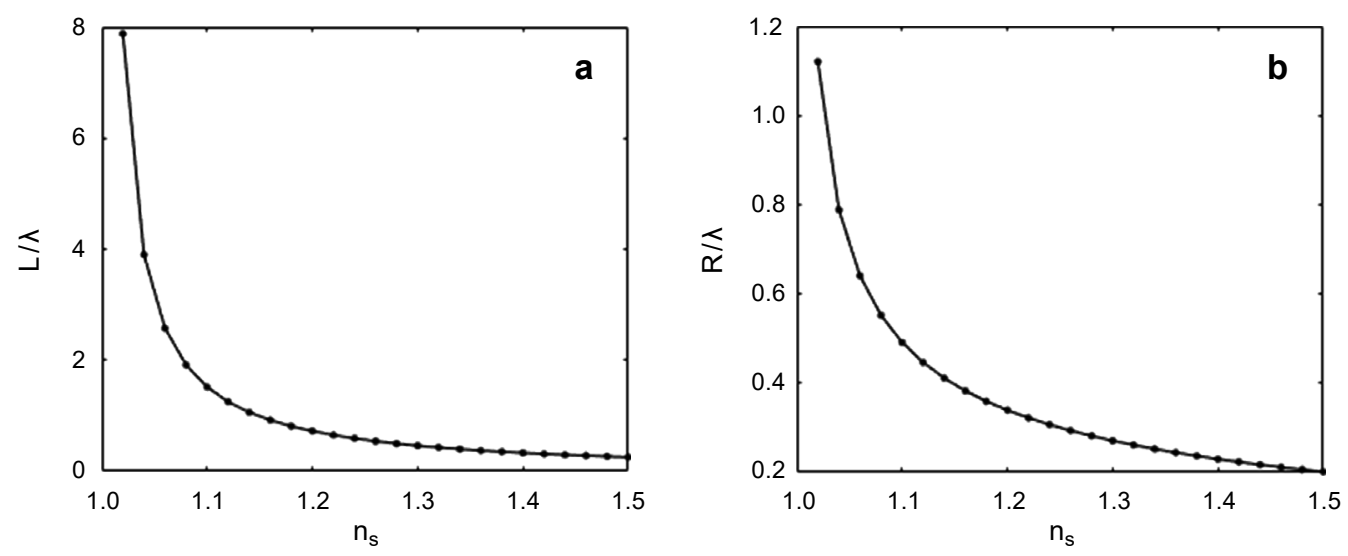

Fig. 2. Convergence properties of the MC algorithm. The maximal length of the thin film layer (in the units of light wavelength) for which the convergence criterion of the MC algorithm is met as a function of its refractive index (a). The maximal radius of the spherical particle (in the units of light wavelength) for which the convergence criterion of the MC algorithm is met as a function of its refractive index (b).

scattering layer or maximum radius of the sphere, for which the algorithm is convergent, drops below the wavelength of the light.

\subsection{Improvement of the Monte Carlo method convergence}

Results presented in Section 2.3 indicate that the convergence of the Neumann expansion is the serious limitation of the presented numerical method. This raises the question of whether the presented method can be improved in order to allow the analysis of light scattering by larger volumes of the scattering medium.

In this section, we present how the procedure similar to preconditioning systems of algebraic equations can be used to improve the convergence properties of the Neumann expansion. The preconditioning problem concerns solving systems of equations in the form of

$$
\mathbf{x}=\mathbf{b}+\mathbf{G x}
$$

where $\mathbf{x}$ is the vector of unknowns, $\mathbf{b}$ is the vector of inputs, and $\mathbf{G}$ is the matrix of coefficients.

Equation (15) can be solved by putting $\mathbf{x}^{(0)}=\mathbf{b}$ and iterating according to the scheme

$$
\mathbf{x}^{(n+1)}=\mathbf{G} \mathbf{x}^{(n)}
$$

The values of $\mathbf{x}^{(n)}$ converge to the solution of Eq. (15) if the spectral radius of the matrix $\mathbf{G}$ is smaller than $1[\underline{17}, \underline{23}]$. If this condition is not met, the system of equations (15) can be transformed into the system

$$
\mathbf{x}=\mathbf{b}^{\prime}+\mathbf{G}^{\prime} \mathbf{x}
$$


which has the same solution as the system (15) but fulfills the convergence criterion. This procedure is called the preconditioning of the linear system [23] . One of the simplest preconditioning procedure is used by the Jacobi iteration scheme. It consists of splitting the matrix $\mathbf{G}$ into its diagonal and non-diagonal parts:

$$
\mathbf{G}=\mathbf{D}+\mathbf{E}
$$

where matrix $\mathbf{D}$ is the diagonal part of the matrix $\mathbf{G}$.

Inserting Eq. (18) into Eq. (15) leads to the new linear system

$$
\mathbf{x}=(\mathbf{I}-\mathbf{D})^{-1} \mathbf{b}+(\mathbf{I}-\mathbf{D})^{-1} \mathbf{E x}
$$

which may have better convergence properties.

Now we adopt this procedure to the one-dimensional integral from Eq. (3). We start by splitting the integral in the right-hand side of the equation into two parts:

$$
E(x)=E^{\mathrm{inc}}(x)+\int_{-\infty}^{\infty} G^{\prime}\left(x, x^{\prime}\right) U\left(x^{\prime}\right) E\left(x^{\prime}\right) \mathrm{d} x^{\prime}+\int_{x-w / 2}^{x+w / 2} G\left(x, x^{\prime}\right) U\left(x^{\prime}\right) E\left(x^{\prime}\right) \mathrm{d} x^{\prime}
$$

where

$$
G^{\prime}\left(x, x^{\prime}\right)= \begin{cases}0 & \text { for } x^{\prime} \in\left(x-\frac{w}{2}, x+\frac{w}{2}\right) \\ G\left(x, x^{\prime}\right) & \text { otherwise }\end{cases}
$$

If $w$ is small enough, values of $E\left(x^{\prime}\right)$ in the entire region $x^{\prime} \in(x-w / 2, x+w / 2)$ can be approximated by $E(x)$. This leads to the equation

$$
(1-d) E(x)=E^{\mathrm{inc}}(x)+\int_{-\infty}^{\infty} G^{\prime}\left(x, x^{\prime}\right) U\left(x^{\prime}\right) E\left(x^{\prime}\right) \mathrm{d} x^{\prime}
$$

where

$$
d=\int_{x-w / 2}^{x+w / 2} G\left(x, x^{\prime}\right) U\left(x^{\prime}\right) \mathrm{d} x^{\prime}
$$

Finally one can obtain the preconditioned integral equation

$$
E(x)=(1-d)^{-1} E^{\mathrm{inc}}(x)+(1-d)^{-1} \int_{-\infty}^{\infty} G^{\prime}\left(x, x^{\prime}\right) U\left(x^{\prime}\right) E\left(x^{\prime}\right) \mathrm{d} x^{\prime}
$$

which has the same form as Eq. (3) and thus can be solved using the Monte Carlo algorithm.

A similar procedure can be applied to the three-dimensional case. The right-hand side integral in Eq. (5) can be split into two parts: 


$$
\mathbf{E}(\mathbf{r})=\mathbf{E}^{\mathrm{inc}}(\mathbf{r})+\int_{V / V_{0}} \overleftrightarrow{\mathbf{G}}\left(\mathbf{r}, \mathbf{r}^{\prime}\right) U\left(\mathbf{r}^{\prime}\right) \mathbf{E}\left(\mathbf{r}^{\prime}\right) \mathrm{d}^{3} \mathbf{r}^{\prime}+\int_{V_{0}} \overleftrightarrow{\mathbf{G}}\left(\mathbf{r}, \mathbf{r}^{\prime}\right) U\left(\mathbf{r}^{\prime}\right) \mathbf{E}\left(\mathbf{r}^{\prime}\right) \mathrm{d}^{3} \mathbf{r}^{\prime}
$$

where $V_{0}$ is the small spherical volume surrounding point $\mathbf{r}$ such that $\left|\mathbf{r}-\mathbf{r}^{\prime}\right|<w / 2$. We further denote this volume as the preconditioning sphere. Again, after the assumption that $\mathbf{E}\left(\mathbf{r}^{\prime}\right) \approx \mathbf{E}(\mathbf{r})$ over the entire preconditioning sphere, one can obtain the preconditioned integral equation

$$
\mathbf{E}(\mathbf{r})=(1-d)^{-1} \mathbf{E}^{\mathrm{inc}}(\mathbf{r})+(1-d)^{-1} \int_{V / V_{0}} \overleftrightarrow{\mathbf{G}}\left(\mathbf{r}, \mathbf{r}^{\prime}\right) U\left(\mathbf{r}^{\prime}\right) \mathbf{E}\left(\mathbf{r}^{\prime}\right) \mathrm{d}^{3} \mathbf{r}^{\prime}
$$

where

$$
d=\int_{V_{0}} \overleftrightarrow{\mathbf{G}}\left(\mathbf{r}, \mathbf{r}^{\prime}\right) U\left(\mathbf{r}^{\prime}\right) \mathrm{d}^{3} \mathbf{r}^{\prime}=U(\mathbf{r})(M-L)
$$

for

$$
M=\frac{8}{3} \pi\left(\left(1-i k \frac{w}{2}\right) \exp \left(i k \frac{w}{2}\right)-1\right)
$$

and

$$
L=\frac{4}{3} \pi
$$

For the derivation of Eq. (27), see papers $[\underline{1}, \underline{14}]$.

\section{Numerical experiments}

In order to analyze the properties of the MC algorithm, we performed two groups of numerical experiments concerning one- and three-dimensional scattering problems. For one-dimensional problems, we used the MC algorithm to compute total electric field distribution for an infinite thin dielectric film illuminated perpendicularly by a planar electromagnetic wave. For three-dimensional problems we used the MC algorithm to compute far-field distribution of the planar electromagnetic wave scattered by the spherical particle. Since for both problems, the known analytical results exist, they can serve as the verification method for the proposed MC algorithm.

\subsection{Verification of Monte Carlo algorithm convergence in the 1D simulations}

In order to verify the convergence properties of the MC algorithm in one-dimensional computations, we simulated the scattering of the electromagnetic wave by a thin dielectric film with varying refractive indices and thicknesses. The mean square difference 
between the electric field distribution computed with the MC method and the analytical solutions were computed. Computations were performed for different values of the maximal path length in the $\mathrm{MC}$ algorithm. If the algorithm converges, the mean square difference should decrease with increased values of the maximal path length. For every
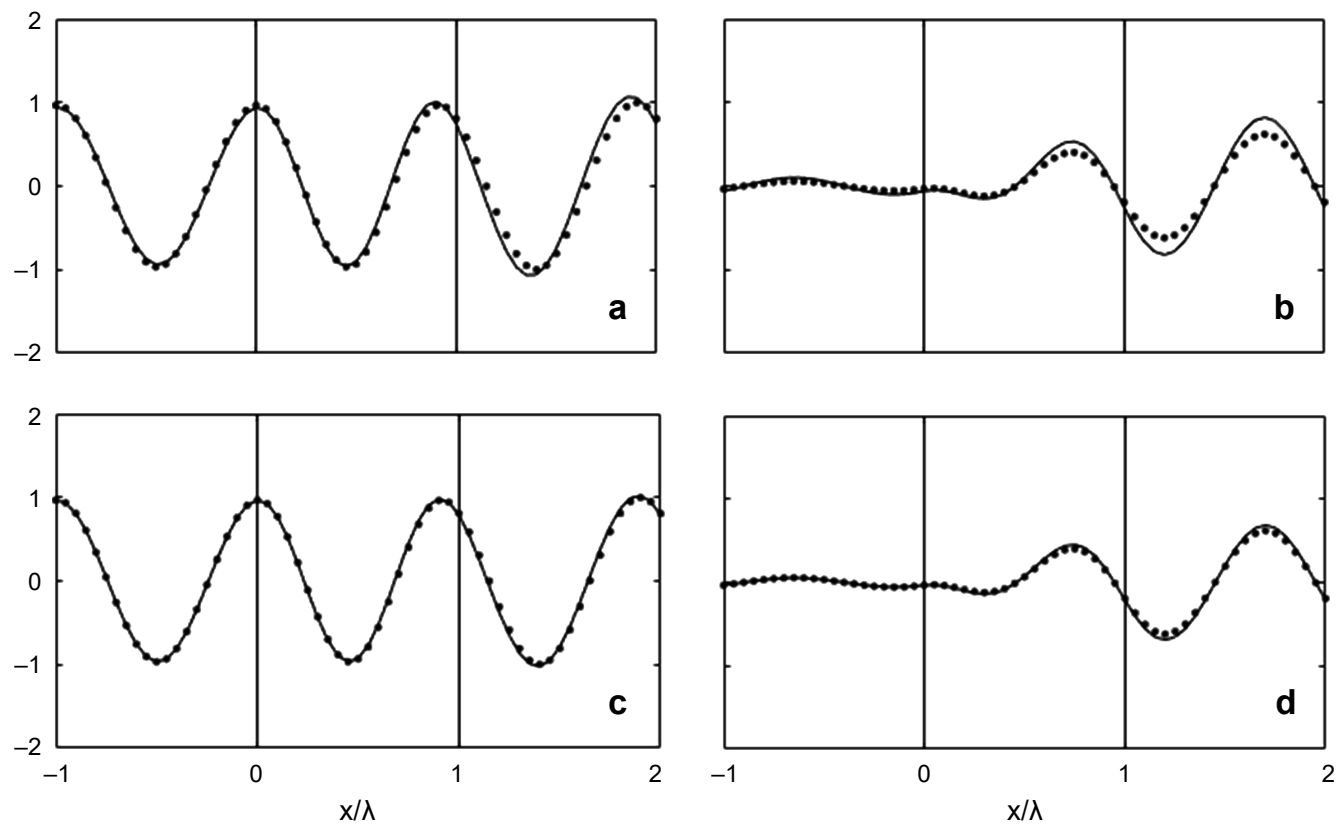

Fig. 3. Example of MC algorithm results for 1D simulations of light scattering by the thin film with refractive index 1.1 and thickness equal to the light wavelength $\lambda$ in a vacuum. The results show real part of the complex amplitude of total field (a and $\mathbf{c}$ ) and scattered field only (b and $\mathbf{d}$ ) for the MC algorithm with maximal path length equal to 3 (a and b) and 10 (c and d). The MC results (dots) are compared with the analytical solution (solid lines).

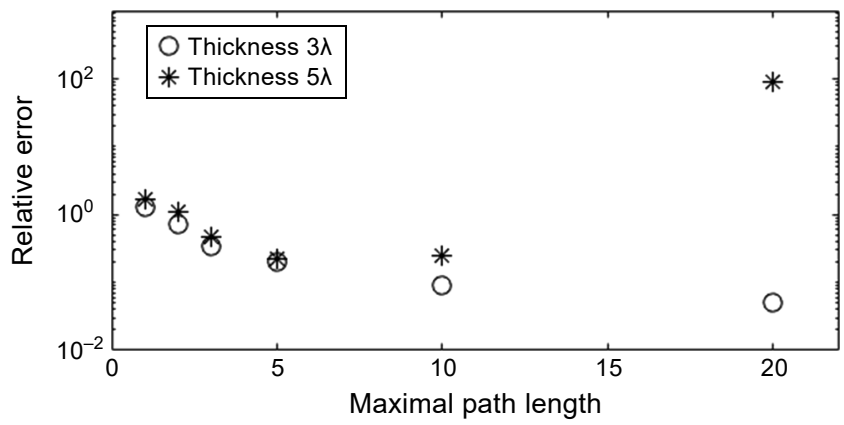

a

Fig. 4. Results of MC computations of the scattered field for 1D light scattering simulations. The computations were performed for thin films with the refractive index equal to 1.05 (a) and 1.33 (b) with varying film thickness and maximal path length in the $\mathrm{MC}$ algorithm. The error was defined as the root mean square difference between the scattered field computed by the MC algorithm and the analytical solution divided by the root mean square value of the analytical solution. 


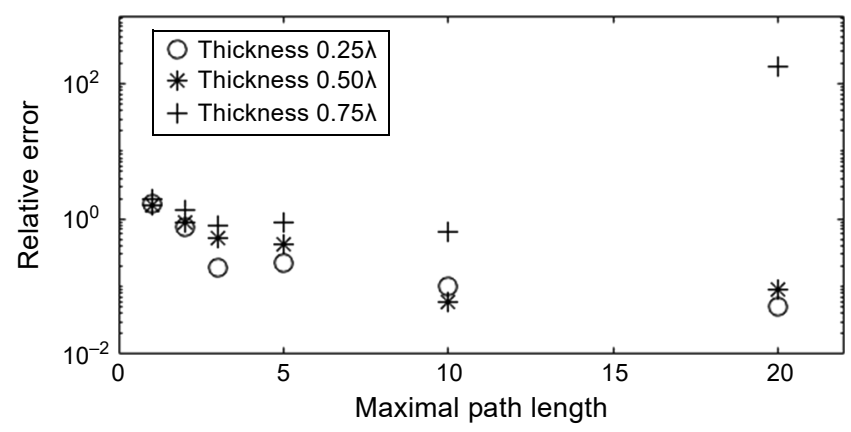

b

Fig. 4. Continued.

simulation, $10^{6}$ iterations of the MC algorithm were used. The example of electric field distribution computed with the MC algorithm compared with the analytical solution is presented in Fig. 3. The results of the computations are presented in Fig. 4.

\subsection{Verification of the preconditioning method in case of $1 D$ simulations}

In order to verify the preconditioning method, the one-dimensional simulations were performed for two films: $i$ ) with refractive index 1.1 and thickness 1.8 of the light wave-
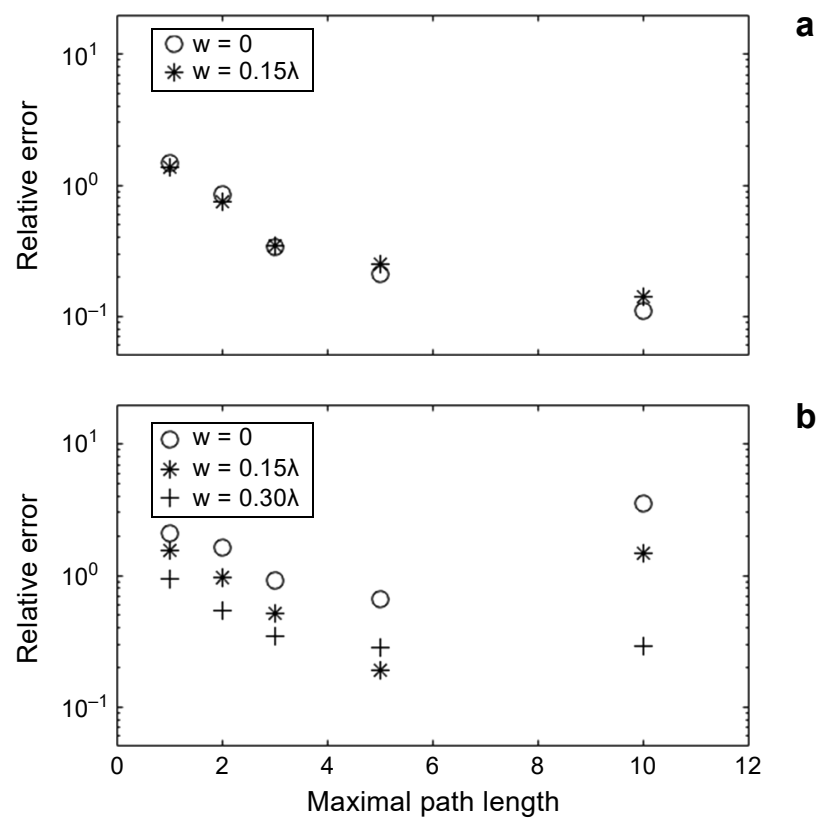

Fig. 5. Results of preconditioned MC computations of the scattered field for 1D light scattering simulations. The computations were performed for two thin films: with the refractive index equal to 1.1 and the thickness equal to $1.8 \lambda$ (a) and with the refractive index equal to 1.2 and the thickness equal to $1.5 \lambda$ (b). The error was defined as the root means square difference between the scattered field computed by MC algorithm and the analytical solution divided by the root mean square value of the analytical solution. 
length and $i$ ) with refractive index 1.2 and thickness 1.5 of the light wavelength. In both cases, simulations were performed for varying maximal path lengths and widths of the precondition window. The number of MC iterations was set to $10^{7}$. Computation results are presented in Fig. 5.

\subsection{Verification of Monte Carlo algorithm convergence in the 3D simulations}

In order to verify convergence of the $\mathrm{MC}$ algorithm for three-dimensional computations, far-field distribution of the intensity of a plane wave scattered by the spherical dielectric particle was computed. Computations were performed for particles with various refractive indices and diameters. For every computation, the $\mathrm{MC}$ algorithm with $10^{6}$ iterations and the diameter of the preconditioning sphere equal to 0.05 of light wavelength were used. The normalized far-field distribution of the scattered wave was computed in the so-called scattering plane, i.e. the plane containing wave vectors of both incident and scattered waves for scattering angles varying from $0^{\circ}$ to $180^{\circ}$. Intensities for two orthogonal states of polarization of the incident wave were analyzed and results were averaged.
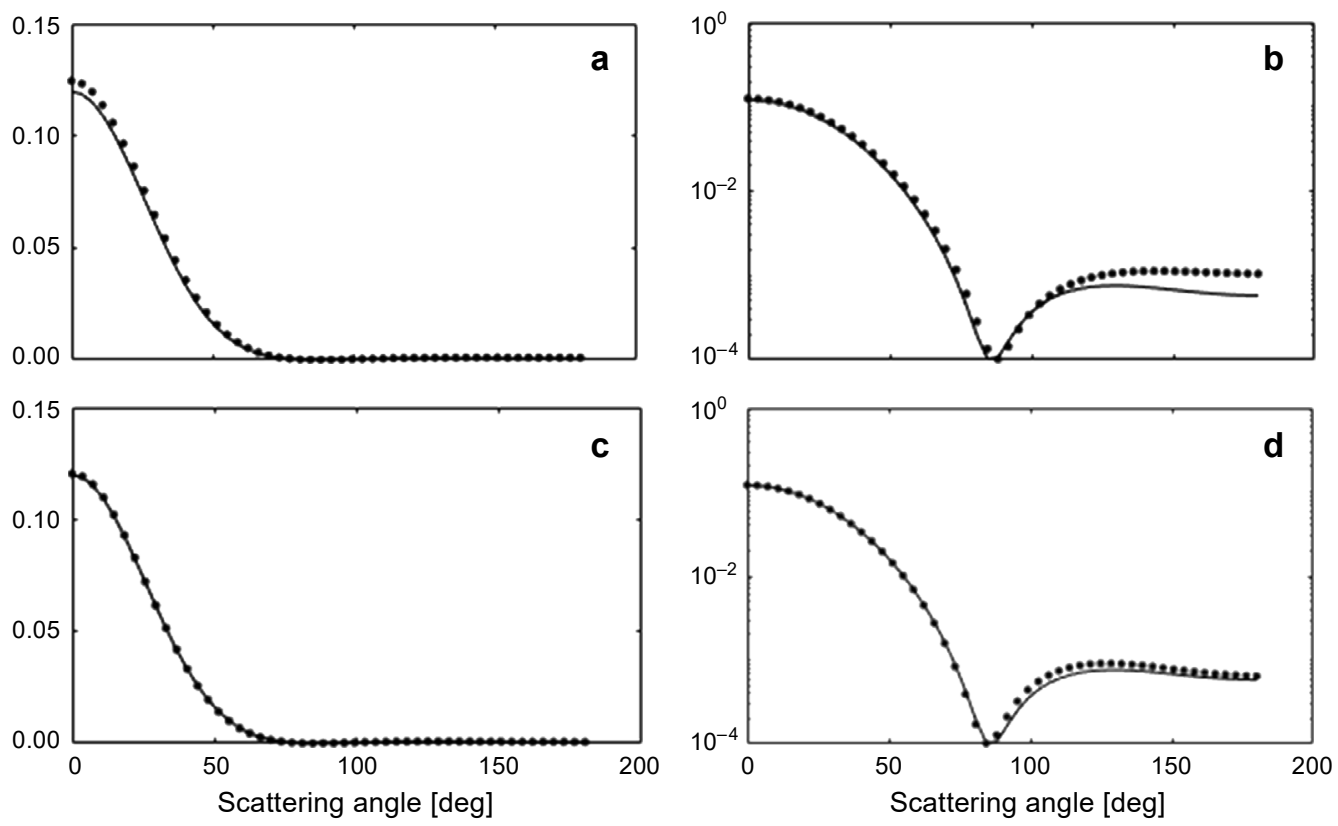

Fig. 6. Examples of MC computation of normalized far-field intensity of the scattered wave. Charts present square values of normalized electric field amplitude in the far-field zone computed for the spherical particle with refractive index 1.1 and diameter equal to the wavelength of the light. The MC results (dots) are compared with the analytical solution (solid lines). Maximal path length used by the MC algorithm was set to 1 (a and $\mathbf{b})$ and 3 (c and $\mathbf{d}$ ). The diameter of the preconditioning sphere was set to $0.15 \lambda$. 


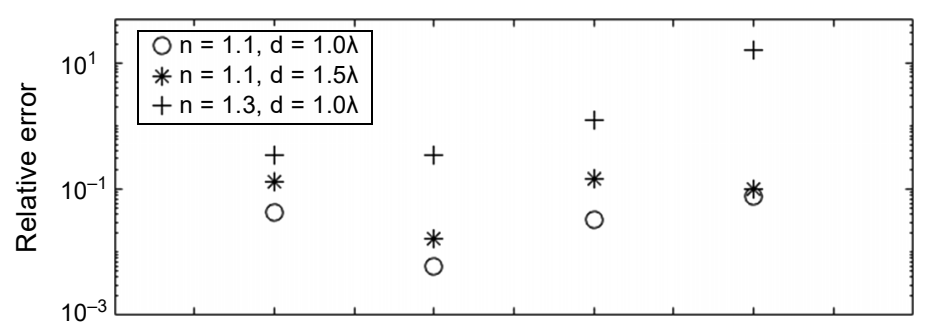

a

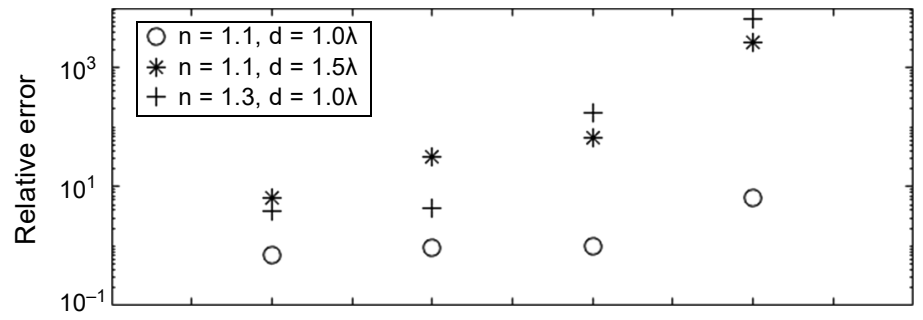

b

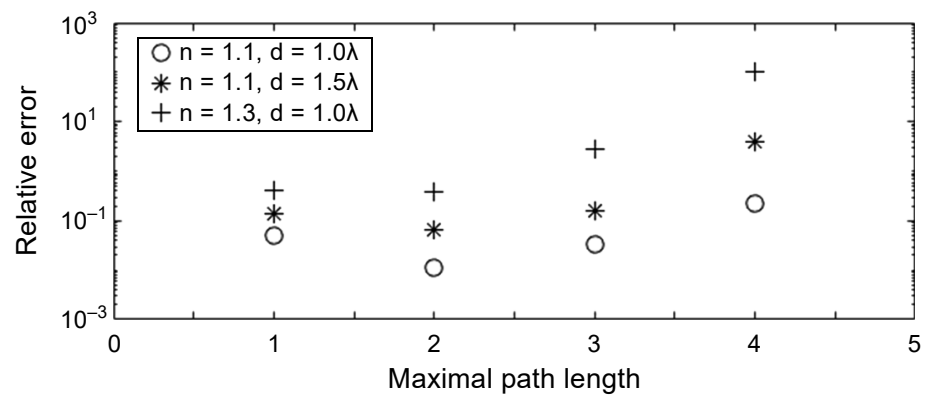

C

Fig. 7. Convergence properties of the $\mathrm{MC}$ algorithm for 3D light scattering simulations. Charts present the relative difference between the normalized intensity of the electric field scattered by the spherical particle in the far-field zone between MC computations and analytical solution. Errors were computed for scattering at angle $0^{\circ}(\mathbf{a}), 180^{\circ}(\mathbf{b})$, and for a root mean square value for all range of scattering angles (c). Various refractive indices $n$ and diameters of the scattering sphere $d$, as well as various maximal path lengths of the MC algorithm, were used.

The results were compared with the analytical solutions by computing the relative difference for scattered wave intensities for scattering angle $0^{\circ}$, for scattering angle $180^{\circ}$ and mean square intensity for all range of scattering angles. Examples of distributions of the scattered electromagnetic wave compared with the analytical solutions are presented in Fig. 6. Computations results are presented in Fig. 7.

\subsection{Verification of the preconditioning method in case of 3D simulations}

In order to verify the proposed preconditioning methods for the $\mathrm{MC}$ algorithm in case of 3D simulation, the far-field distribution of the light scattered by a spherical particle 
was computed. Computations were performed for three particles: $i$ ) with refractive index 1.1 and diameter equal to $1 \lambda, i i$ ) with refractive index 1.1 and diameter equal to $1.5 \lambda$, and $i i i)$ with refractive index 1.3 and diameter equal to $1 \lambda$. All computations were performed using the $\mathrm{MC}$ algorithm with $10^{7}$ iterations. Maximal path length was varying from 1 to 4 . Computations were repeated for the diameter of the preconditioning sphere equal to $0.05 \lambda, 0.15 \lambda$, and $0.3 \lambda$. In each case, the angular distribution of the normalized intensity of the electric field in the far-field was computed and compared with the analytical solution. Average values for two orthogonal states of polarization were taken for computations. The relative difference of the scattered wave intensity for scattering at angle $0^{\circ}$ and $180^{\circ}$ were computed as well as the relative difference of the scattered

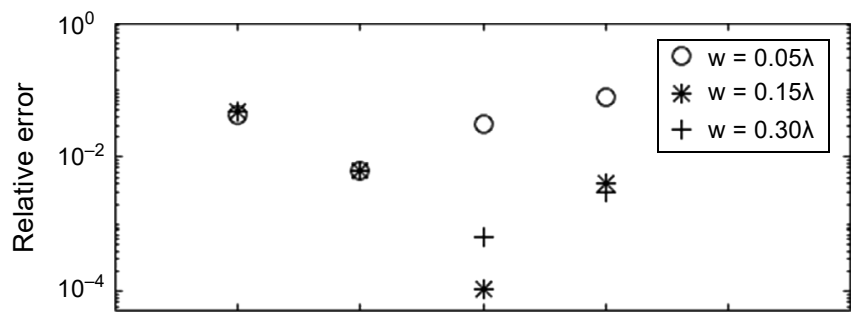

a

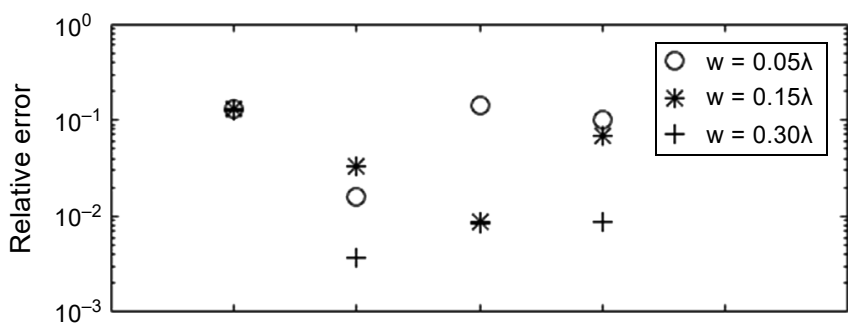

b

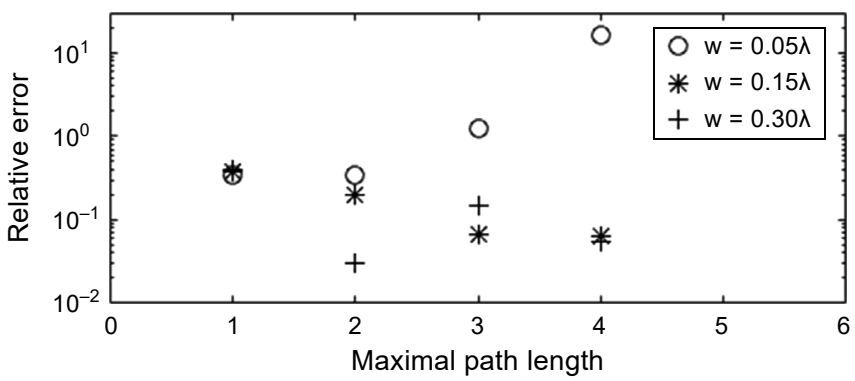

C

Fig. 8. Comparison of $\mathrm{MC}$ computations for scattering angle equal to $0^{\circ}$. Charts present the relative differences between the normalized intensity of the scattered wave in the far-field zone computed with the $\mathrm{MC}$ algorithm and analytical solution. Computations were performed for three types of spherical particles: with refractive index 1.1 and diameter $1 \lambda$ (a), with refractive index 1.1 and diameter $1.5 \lambda(\mathbf{b})$, and with refractive index 1.2 and diameter $1 \lambda(\mathbf{c})$. The diameter of the preconditioning window $w$ was varying from $0.05 \lambda$ to $0.3 \lambda$. 


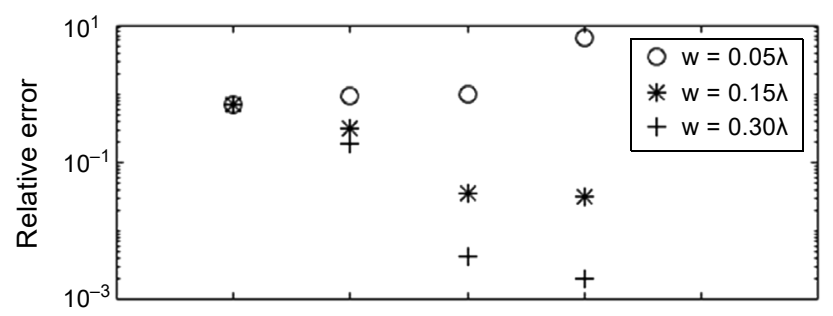

a

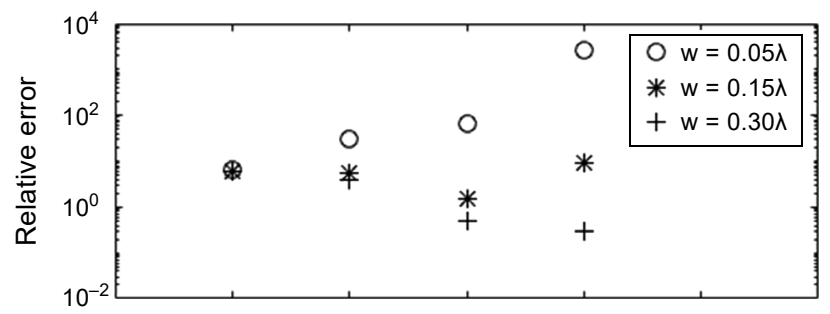

b

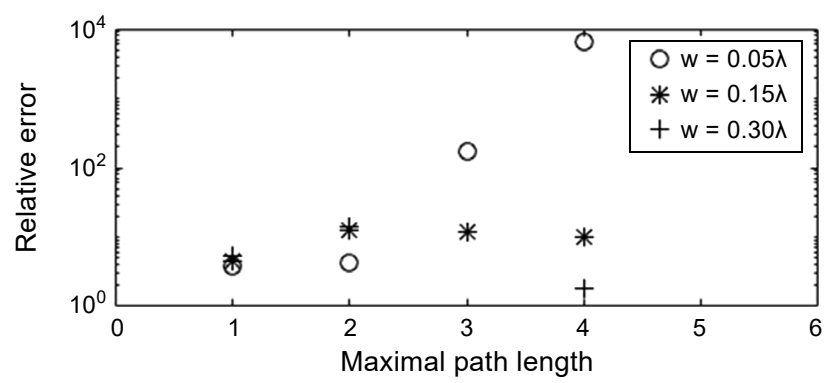

C

Fig. 9. Comparison of $\mathrm{MC}$ computations for scattering angle equal to $180^{\circ}$. Charts present the relative differences between the normalized intensity of the scattered wave in the far-field zone computed with the MC algorithm and analytical solution. Computations were performed for three types of spherical particles: with refractive index 1.1 and diameter $1 \lambda$ (a), with refractive index 1.1 and diameter $1.5 \lambda(\mathbf{b})$, and with refractive index 1.2 and diameter $1 \lambda(\mathbf{c})$. The diameter of the preconditioning window $w$ was varying from $0.05 \lambda$ to $0.3 \lambda$.

wave intensity averaged for all range of scattering angles. The values for two states of polarization of the incident wave were computed and averaged. The computation results are presented in Figs. 8-10.

\section{Discussion}

The presented results show that the convergence problem is crucial for applying the presented MC algorithm for light scattering simulations. The method itself allows to achieve very high accuracies of the results (below 1\%) but only for the cases where the maximal path lengths can be limited to the low values. This can be achieved only for scattering objects with small refractive indices and dimensions comparable to the wavelength of the light. 


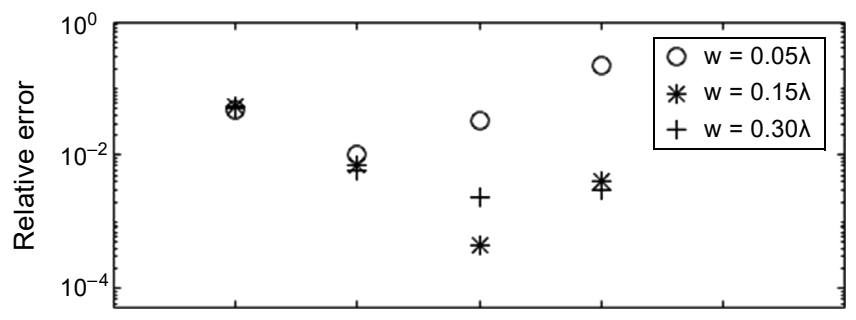

a

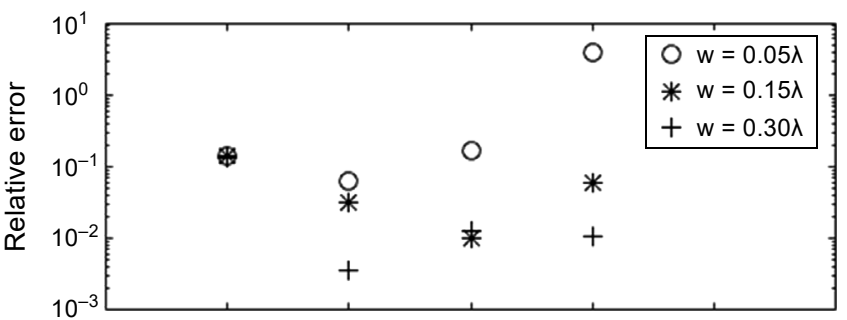

b

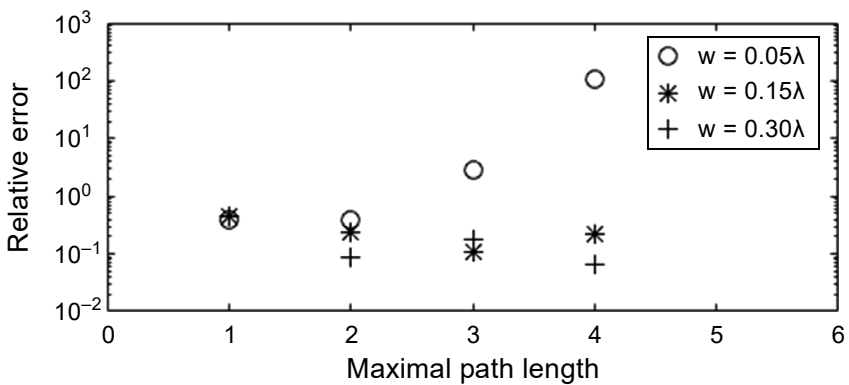

C

Fig. 10. Comparison of MC computations averaged for all scattering angles. Charts present relative difference between the normalized intensity of the scattered wave (averages for scattering angles from $0^{\circ}$ to $180^{\circ}$ ) in the far-field zone computed with the MC algorithm and analytical solution. Computations were performed for three types of spherical particles: with refractive index 1.1 and diameter $1 \lambda$ (a), with refractive index 1.1 and diameter $1.5 \lambda(\mathbf{b})$, and with refractive index 1.2 and diameter $1 \lambda(\mathbf{c})$. The diameter of the preconditioning window $w$ was varying from $0.05 \lambda$ to $0.3 \lambda$.

Comparing the results presented in Section 3 with theoretical predictions of MC algorithm convergence (Fig. 2) shows that the algorithm may be convergent even if the criterion given by Eq. (10) is not fulfilled. This can be attributed either to the fact that Eq. (10) provides a stronger criterion than limiting the spectral radius of an integral operator or the fact that maximal path length in $\mathrm{MC}$ algorithm implementation was limited to a small value. Equation (10) is still useful for providing rough estimation for the limit of MC algorithm applicability.

The presented preconditioning method can improve the convergence of the MC algorithm but only with moderate results. This is caused by the fact that the method is based on the assumption that the electric field in a certain volume of the scattering object is constant. This assumption is valid if the preconditioning volume is significantly smaller than the wavelength of the light. On the other hand, limiting the size of the preconditioning volume reduces its impact on the algorithm convergence. 
The preconditioning method seems to provide more improvement for three-dimensional computations that for the one-dimensional ones. This may be attributed to the fact that the amplitude of the three-dimensional Green's function decreases with the distance between the two points and the preconditioning method excludes a part of the integral operator where its amplitude is relatively strong. This limits the norm of integral operation more efficiently than for one-dimensional case where the amplitude of Green's function remains constant.

An interesting fact is that the $\mathrm{MC}$ algorithm in the three-dimensional case can provide very accurate results even in case of a large preconditioning volume. For some computations even for volumes with the diameter equal to 0.3 of the wavelength accuracies better than $1 \%$ were obtained. On the one hand, the preconditioning is based on the assumption of the electric field being constant inside the preconditioning sphere what should limit the accuracy of the MC algorithm, but on the other hand, even with preconditioning, the $\mathrm{MC}$ algorithm is still not based on the stiff discretization of the scattering object since the preconditioning volume changes in every iteration.

It is much more difficult to obtain accurate results in case of backward scattering where electric field intensity is smaller than in case of forward scattering. In case of the scattering sphere with diameter $1 \lambda$ and refractive index 1.1 , the preconditioning method allows to obtain accuracy below $1 \%$ for scattering angle equal to $180^{\circ}$ but for larger spheres or refractive indices, the obtained accuracies were much worse.

All the discussed problems show that even though the presented $\mathrm{MC}$ algorithm is certainly valid and allows to obtain highly accurate results if implemented properly, its class of applications is limited to small variations of refractive index and small volumes of the scattering objects. It makes claims that the $\mathrm{MC}$ algorithm can be applied to the macroscopic volumes of the scattering media such as biological tissues dubious.

The question remains whether this situation can be improved. Perhaps the development of better preconditioning methods might lead to an increase of the $\mathrm{MC}$ algorithm convergence and extends its range of applications.

It is also important to notice that the presented study does not concern the variance of the MC algorithm, i.e. the relation between the accuracy and number of MC iterations. For this reason, the large numbers of $\mathrm{MC}$ iterations were used to generate the presented results. However, there are methods of improving the $\mathrm{MC}$ algorithm variance, e.g. the usage of the Reversible-Jump Markov Chain Monte Carlo algorithm suggested in [19]. Therefore, the number of iterations used in this study should be possible to reduce.

\section{Conclusions}

We implemented the $\mathrm{MC}$ algorithm for solving the volume integral equation describing the light scattering process. We show that the algorithm is valid and can provide accurate results. However, due to its convergence properties, its range of applications is limited to the small variations of the refractive index and small volumes of the scattering objects. We also showed the preconditioning method that allows us to improve 
the convergence of the $\mathrm{MC}$ algorithm but only with the moderate effect. Applying the $\mathrm{MC}$ algorithm for light scattering simulations in large volumes of the scattering objects requires the development of different approaches, e.g. by applying more sophisticated preconditioning methods.

Acknowledgments - This research work has been supported by The National Science Centre, Poland under grant No. 2014/15/N/ST7/04903 and DS program of Faculty of Electronics, Telecommunications and Informatics, Gdańsk University of Technology.

\section{References}

[1] Taflove A., Hagness S.C., Computational Electrodynamics: The Finite-Difference Time-Domain Method, 3rd Ed., Artech House, Norwood, MA, 2005.

[2] LakHTAKia A., Strong and weak forms of the method of moments and the coupled dipole method for scattering of time-harmonic electromagnetic fields, International Journal of Modern Physics C 3(3), 1992, pp. 583-603, DOI: 10.1142/S0129183192000385.

[3] Yurkin M.A., HoeKstRa A.G., The discrete dipole approximation: an overview and recent developments, Journal of Quantitative Spectroscopy and Radiative Transfer 106(1-3), 2007, pp. 558-589, DOI: 10.1016/j.jqsrt.2007.01.034.

[4] Mishchenko M.I., Electromagnetic Scattering by Particles and Particle Groups: An Introduction, Cambridge University Press, Cambridge, 2014.

[5] Draine B.T., Flatau P.J., Discrete-dipole approximation for scattering calculations, Journal of the Optical Society of America A 11(4), 1994, pp. 1491-1499, DOI: 10.1364/JOSAA.11.001491.

[6] Mischchenko M.I., Travis L.D., MAckowski D.W., T-matrix computations of light scattering by nonspherical particles: a review, Journal of Quantitative Spectroscopy and Radiative Transfer 55(5), 1996, pp. 535-575, DOI: 10.1016/0022-4073(96)00002-7.

[7] Martelli G.Z.F., Del Bainco S., Ismaelli A., Light Propagation Through Biological Tissue and Other Diffusive Media: Theory, Solutions and Software, SPIE Press Monograph, Vol. PM193, Bellingham, 2010.

[8] WANG L., JACQUes S.L., Zheng L., MCML - Monte Carlo modeling oflight transport in multi-layered tissues, Computer Methods and Programs in Biomedicine 47(2), 1995, pp. 131-146, DOI: $\underline{10.1016 /}$ 0169-2607(95)01640-F.

[9] Doronin A., Radosevich A.J., Backman V., Meglinski I., Two electric field Monte Carlo models of coherent backscattering of polarized light, Journal of the Optical Society of America A 31(11), 2014, pp. 2394-2400, DOI: 10.1364/JOSAA.31.002394.

[10] Smithies D.J., Lindmo T., Chen Z., Nelson J.S., Milner T.E., Signal attenuation and localization in optical coherence tomography studied by Monte Carlo simulation, Physics in Medicine and Biology 43(10), 1998, pp. 3025-3044, DOI: 10.1088/0031-9155/43/10/024.

[11] PLUCIŃSKi J., FRYDRYCHOWSKI A.F., New aspects in assessment of changes in width of subarachnoid space with near-infrared transillumination/backscattering sounding, part 1: Monte Carlo numerical modeling, Journal of Biomedical Optics 12(4), 2007, article 044015, DOI: 10.1117/1.2757603.

[12] Pluciński J., Frydrychowski A.F., Influence of pulse waves on the transmission of near-infrared radiation in outer-head tissue layers, Frontiers of Optoelectronics 10(3), 2017, pp. 287-291, DOI: 10.1007/s12200-017-0723-7.

[13] Kraszewski M., Pluciński J., Application of the Monte Carlo algorithm for solving volume integral equation in light scattering simulations, Optica Applicata 50(1), 2020, pp. 1-15, DOI: 10.37190/ oa200101.

[14] Xiong G., Xue P., Wu J., Miao Q., Wang R., Ji L., Particle-fixed Monte Carlo model for optical coherence tomography, Optics Express 13(6), 2005, pp. 2182-2195, DOI: 10.1364/OPEX.13.002182. 
[15] Daria V.R., Saloma C., Kawata S., Excitation with a focused, pulsed optical beam in scattering media: diffraction effects, Applied Optics 39(28), 2000, pp. 5244-5255, DOI: 10.1364/AO.39.005244.

[16] Devaney A.J., Mathematical Foundations of Imaging, Tomography and Wavefield Inversion, Cambridge University Press, Cambridge, 2012.

[17] Dunn W.L., Shultis J.K., Exploring Monte Carlo Methods, Elsevier Science, Amsterdam, 2011.

[18] Doucet A., Johansen A.M., Tadić V.B., On solving integral equations using Markov chain Monte Carlo methods, Applied Mathematics and Computation 216(10), 2010, pp. 2869-2880, DOI: $10.1016 /$ j.amc.2010.03.138.

[19] Pereira P.F., Sherif S.S., Simulation of the interaction of light and tissue in a large volume using a Markov chain Monte Carlo method, Proceedings of SPIE 8412, 2012, article 841218, DOI: 10.1117 I 12.2001455 .

[20] Kraszewski M., Pluciński J., Coherent-wave Monte Carlo method for simulating light propagation in tissue, Proceedings of SPIE 9706, 2016, article 970611, DOI: 10.1117/12.2213213.

[21] Born M., Wolf E., Principles of Optics: Electromagnetic Theory of Propagation, Interference and Diffraction of Light, 7th Ed., Cambridge University Press, New York, 2013.

[22] Akkermans E., Montambaux G., Mesoscopic Physics of Electrons and Photons, Cambridge University Press, Cambridge, 2007.

[23] SAAD Y., Iterative methods for sparse linear systems, 2nd Ed., Society for Industrial and Applied Mathematics, Philadelphia, 2003. 